\section{Editor’s Note}

Through this column, we hope that practitioners in general medical settings will gain a more complete knowledge of the many patients who are likely to benefit from brief psychotherapeutic interventions. A close working relationship between primary care and psychiatry can serve to enhance patient outcome.

Dr. Varon is in the fifth year of a combined psychiatryneurology residency at the Medical University of South Carolina.

\section{Biweekly Cognitive Therapy for Social Phobia}

\author{
Daniel Varon, M.D.
}

$\mathbf{T}$ raditionally, insight-oriented psychotherapy is a once-weekly, 50-minute venture. Cognitive therapy is typically offered at a similar frequency. There is legitimate concern that the continuity of the process would be disrupted by less frequent meetings.

Social phobia is a relatively common disorder. The prevalence of social phobia in primary care settings ranges from $2.9 \%$ to $7.0 \%$. As in community samples, social phobia frequently occurs with other disorders in primary care patients, most commonly major depression, generalized anxiety disorder, and substance abuse. In patients with this disorder, brief therapy has been used successfully alone or in conjunction with medications. The therapy is usually conducted once a week, or sometimes twice a week if necessary, which can be difficult to afford for some patients. The patient described below presented with a case of major depressive disorder with comorbid generalized social phobia and some obsessivecompulsive traits and was seen for 10 brief therapy sessions every other week with significant benefit. This case demonstrates the usefulness of biweekly therapy for a subset of patients in whom weekly therapy is not an option.

\section{PRESENTATION OF THE PROBLEM}

The patient is a 31-year-old single Asian American man who had been treated for 5 months for depression and anxiety with pharmacotherapy at the university clinic. The patient's depressive symptoms had improved, but he continued to experience marked anxiety. He had moved from Rhode Island to Charleston, S.C., 6 months prior to his visit with me (D.V.) in order to start a new job at an insurance company. The patient said he frequently felt anxious, particularly around his boss and female coworkers because he was constantly fearful of saying or doing "the wrong thing" in front of them. He had to give monthly presentations in front of his coworkers, and, although he usually did fine, he would worry about his performance for 2 weeks prior to the presentation. He also had difficulties interacting with people in social situations unless he had a drink or two beforehand. He considered himself a loner, preferring to stay at home watching television or taking care of his car.

The patient also mentioned having a motor vehicle accident soon after moving to Charleston in which his car was badly damaged. He had to purchase a new car due to the accident. He had become very careful with his new car, spending a great deal of time washing it on the weekends, making sure the paint was in good condition every day as he came back from work, and fixing minor scratches as they occurred. He would also drive the car as little as possible to decrease the chances of it getting scratched or damaged, and he would avoid going to places where the parking spaces were too close to each other. He would also spend a good bit of time looking for a parking space at work where the car would be safe from careless drivers. He denied any flashbacks from the accident or 
being concerned about his safety. He thought his care of the car might be a little excessive, but he really enjoyed it and did not think it was impairing his daily activities.

\section{PSYCHOTHERAPY}

During his initial evaluation, the patient made poor eye contact and seemed mildly anxious, but his affect had a full range. His speech was normal, and his thought process was goal-directed with no evidence of depressive, suicidal, or psychotic content. He described his symptoms of depression, including low energy, decreased appetite, poor sleep, and decreased motivation, all of which had improved after 3 months of pharmacologic treatment. However, his symptoms of anxiety were unchanged. My (DSM-IV) diagnostic impressions included generalized social phobia (with evidence of persistent fear of social situations or performance in public, avoidance of social interaction, and anticipatory anxiety) and major depressive disorder in full remission (during the past 2 months, no significant signs or symptoms of the disturbance were present). The patient demonstrated some obsessive-compulsive traits, but did not meet criteria for obsessive-compulsive disorder or obsessive-compulsive personality disorder. He felt comfortable on the medication regimen he was taking at the time, which included paroxetine and bupropion. We discussed other options for treatment, and I briefly reviewed the cognitive model with him. I explained that we needed to start by identifying "automatic thoughts," which in turn could affect the way he felt about certain situations. We agreed to meet for 10 sessions initially and to review our progress around session 6 . He was not able to afford weekly sessions, so I agreed to see him once every 2 weeks.

During our second session, I discussed the cognitive model in more depth, and we started by looking at situations that made him uncomfortable. He stated that he felt most anxious in the days prior to his work presentations. During this time, he would have thoughts of losing his job, of his coworkers not being able to understand what he was presenting and of being ridiculed, and of his boss thinking he had done a very poor job. These thoughts clearly represented 2 common cognitive errors, catastrophizing and polarization. When asked what evidence he had to support these thoughts, he realized there had not been any times in the past when his boss or coworkers had done what he was concerned about. He was able to refute the thoughts by generating alternative thoughts that included, "If my boss thought I had done a poor job in the past, he would have said so," "I have done well in the past, so therefore this time should be no different," and "I usually prepare well, so I should do fine in the presentation." At the end of the session, we discussed the use of triple columns to record distressful situations, the associated feelings, and thoughts or meanings that accompanied the feelings.

During our third and fourth sessions, we reviewed the triple-column contents, looked for distorted cognitions, and proposed alternative thoughts. We also discussed his anticipatory anxiety prior to presentations in front of an audience, his concerns about talking to his boss, and his fears of ridicule by his coworkers.

During session 5, he commented on the fact that he had been preparing for a presentation and was able to overcome his anxiety and fears of failure. He seemed pleased with the outcome of his talk. He mentioned a situation in which he had gone to a bar the week before with his coworkers. He looked for a parking space for 15 minutes and although he did not feel comfortable with the space he found, he decided to park. He had to leave the place 30 minutes later because he feared his car might have gotten scratched. He also mentioned that he would look for minor alterations in the paint every day after work and would try to have them touched up that same day. I asked what would happen if he waited until the weekend to fix the "defects." He replied, "Probably nothing." We were able to identify several distorted cognitions regarding his car by looking for evidence to support or disprove his thoughts.

We evaluated his progress during session 6 , noting how comfortable he had become with the cognitive model. It clearly made sense to him, and he would employ it frequently when feeling anxious. We continued to work on situations that evoked anxious feelings, including interactions with people in social gatherings and his belief that people noticed his anxiety in these situations.

By session 9, he said he was no longer feeling excessively anxious about presentations at work, discussing issues with coworkers, or approaching his boss. He had substantially decreased the amount of time he spent caring for his car, no longer obsessing about minor issues, and, although he still was careful about parking, he did not spend as much time finding a spot as he used to. He felt that he had made a lot of progress and thought he could continue to apply the cognitive model on his own.

We agreed to meet 4 weeks later to review how he was doing. On his return visit, he reported that he was doing well. He had been on a trip with a friend from college and had attended a wedding, where he had been able to talk to several people with less difficulty than ever before. We agreed to continue to meet every 2 months for medication management only. This case demonstrates that, although ideally brief therapy should be done on a weekly basis, in some instances in which the patient is highly motivated to work, biweekly sessions can be used with success. 